\title{
Effect of Kraft Paper Barriers on Bridging in Contaminated Transformer Oil
}

\author{
Shekhar Mahmud*, Igor O. Golosnoy, George Chen \\ Electronics and Computer Science, \\ University of Southampton, \\ Southampton, SO17 1BJ, UK \\ *E-mail: sm8e08@ecs.soton.ac.uk
}

\begin{abstract}
The paper investigates effects of Kraft Paper to prevent bridging in pressboard fibre contaminated transformer oil. There are three different configuration of Kraft paper tested. These are 1) covered electrode with loose bond, 2) tight bond and 3) bare electrode with a paper barrier in the middle. There were no bridges formed with loosely bonded tests with DC electric fields. For the other two cases, there was always a complete bridge formed.
\end{abstract}

Keywords-dielectrophoresis; bridging; charge transportation; Coulomb force; transformer oil.

\section{INTRODUCTION}

One of the key components in high voltage transmission and distribution systems are power transformers. But a significant amount of total transformer failures are caused by insulation oil. Therefore, it is essential to understand the failure mechanisms so the proper measures can be taken [1].

Transformer oil is in contact with metal, iron core and pressboard insulation. Contaminants such as metal filings or cellulosic residue can be formed in the oil. These contaminants tend to move towards high field regions due to dielectrophoresis (DEP) forces and could form a bridge over a period of time [2]. An important element of the bridging phenomenon is an electric charge accumulation by the particles and their migration under a combination of Coulomb and DEP forces. In fact, the charging is essential to the bridge formation [3]. To prevent the contaminant particles contacts with the transformer parts (and reduction in the particle charging) pressboard barriers are used along with oil in a transformer. Previously we have reported the bridging with bare spherical electrode under different combinations AC and DC electric fields as well as mathematical model of the bridging [3-7]. This paper explores the effectiveness of the paper barrier in terms of stopping the bridge formation.

Three types of experiments have been carried out to investigate the effect of kraft paper barrier on particle accumulation between two electrodes with different potentials under several levels of AC and DC voltages. Spherical electrodes were covered with 100 microns thick kraft paper with loose or tight bond. In another experiment bare electrodes were used and a kraft paper barrier is placed in oil in the middistance between them.

\author{
Gordon Wilson and Paul Jarman \\ National Grid \\ Warwick Technology Park \\ Warwick, UK
}

\section{EXPERIMENTS}

\section{A. Sample Tank}

The sample tank used for all the experiments was glass built with a volume of $550 \mathrm{ml}$. A pair of spherical electrodes with a diameter of $13 \mathrm{~mm}$ have used for the experiments. The electrodes were made of brass. The electrodes were attached to either side of sample tank wall from which they extended towards the middle of the cell. The electrodes' gap was kept constant at $10 \mathrm{~mm}$.

\section{B. Sample Preparations}

Pressboard fibre was produced by rubbing a piece of new pressboard which normally used in high voltage transformer with different sizes of metal files. There were four different sizes of sieves were used to separate the fibres. Only one size of particles was used for these experiments which is 150-250 $\mu \mathrm{m}$. The fibres were separated by their width rather than length. The contamination levels tested for loose bonded electrode were $0.001 \%, 0.002 \%, 0.003 \%, 0.004 \%, 0.006 \%$, $0.008 \%$ and $0.016 \%$ by weight. For the paper barrier test, only $0.001 \%$ to $0.003 \%$ contaminants were investigated whereas for tight bonded electrode only $0.008 \%$ used.

The sample tank was cleaned with a soap solution in hot water then it was dried in hot air flow before starting a new test with a new size of particle. For repeating a test with same particle size, the sample tank was first rinsed with some clean oil then the test cell was rinsed thoroughly with cyclohexene.

\section{Experimental Setup}

The sample tank was positioned under a stereo microscope that had a digital camera mounted on the top to record the optical images of the particle accumulation. For experiments with DC electric field, the microscope along with the test cell was placed inside an aluminium box which acts as a Faraday Cage as the conduction current was too noisy. A desktop computer was used to control the digital camera and also store data from camera, Keithley Picoammeter 6485 (DC) for conduction current measurement. A block diagram of complete experimental setup for DC experiments is shown in Fig. 1. A detail description of DC experiments can be found in [5]. A new piece of $100 \mu \mathrm{m}$ thick kraft paper used to cover the surface of the both electrodes. A single layer of paper was wrapped around the electrodes and were kept without tied to 
rod attached to electrodes which was the case of loose bonded test. The second test was conducted with a new single layer of paper wrapped around the electrodes as well as it was tied tightly to the rod of the electrodes with cable tie. The third set of experiments were performed with the paper barrier placing the mid-point of the electrodes as shown in Fig. 2. The barrier was wider than the diameter of the electrodes. The barrier was held in the middle from either side of electrode with plastic foam.

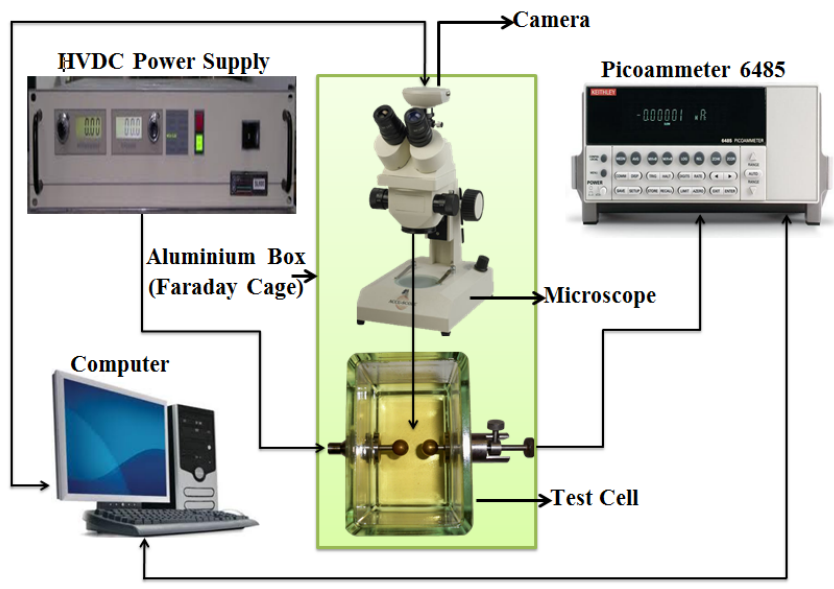

Fig. 1. Complete block diagram of experimental setup under influence of DC electric field [5].

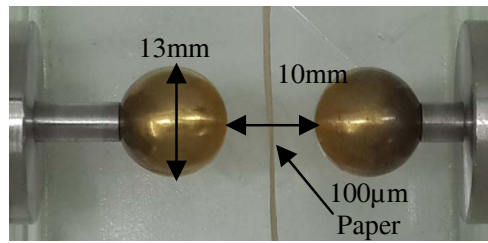

Fig. 2. Spherical electrodes with $100 \mu \mathrm{m}$ kraft paper barrier in the middle.

\section{Experiments under different electric field}

The DC high voltage (HV) source was connected to one of the spherical electrodes. The other electrode was connected to a Keithley picoammeter 6485 (DC) to measure the conduction current through the gap and it was finally connected to load return of the HV source. Three different voltage levels were investigated for each category of contamination levels, such as DC $(2 \mathrm{kV}, 7.5 \mathrm{kV}$ and $15 \mathrm{kV})$ for loose bonded electrode and Kraft paper barrier test and DC $(1 \mathrm{kV}, 3 \mathrm{kV}$ and $6 \mathrm{kV})$ for tight bonded test. All the experiments were carried out until a complete bridge was created between the electrodes or maximum of 25 minutes where there was no bridge. Optical microscopic images were also taken in a regular interval during the entire test to record the bridging process. All the experiments were conducted at ambient room temperature. The temperature of the aluminium box was monitored during the experiments. The temperature of the room was controlled and was within 3 degrees difference for all the DC experiments. The experiments were repeated three times for each voltage level to observe the repeatability of the results.

\section{RESULTS AND DISCUSSION}

\section{A. Bridging with loose bond electrode}

Fig. 3 shows the images of particle accumulation process for the loosely bonded covered electrodes test. The contaminated pressboard fibres were started to move slowly towards both electrodes as soon as $2 \mathrm{kV}$ supply was turned on. This movement were due to DEP force drawing the particles near to high gradient of electric field. They started to stick themselves to the surface of the electrodes. The particles were aligned themselves parallel to electric field lines. The particles were covering the electrode surfaces until $20 \mathrm{~min}$ of the test whereby there was not many particles left near the vicinity of the electrodes.

The movement of the particles were faster with the applied voltage of $7.5 \mathrm{kV}$. This time particles were sticking to the electrode surfaces with greater speed. A shallow beard like cover of pressboard particles surrounded the electrodes.

After the $15 \mathrm{kV}$ applied the sample, the particles were violently moving close to the surface of the electrodes. Within $1 \mathrm{~min}$ they covered most of the electrode surfaces. The fibre beard cover became thicker with time up to $20 \mathrm{~min}$. None of the voltage levels tested were able to create a bridge between the electrodes. The oil layer beneath the kraft paper prevented charge transportation to the other side of the paper surface. So the particles could not get enough charge to detach themselves from the surface to go back and forth as observed for the case of bare electrode test as reported previously by us [5 - 10].

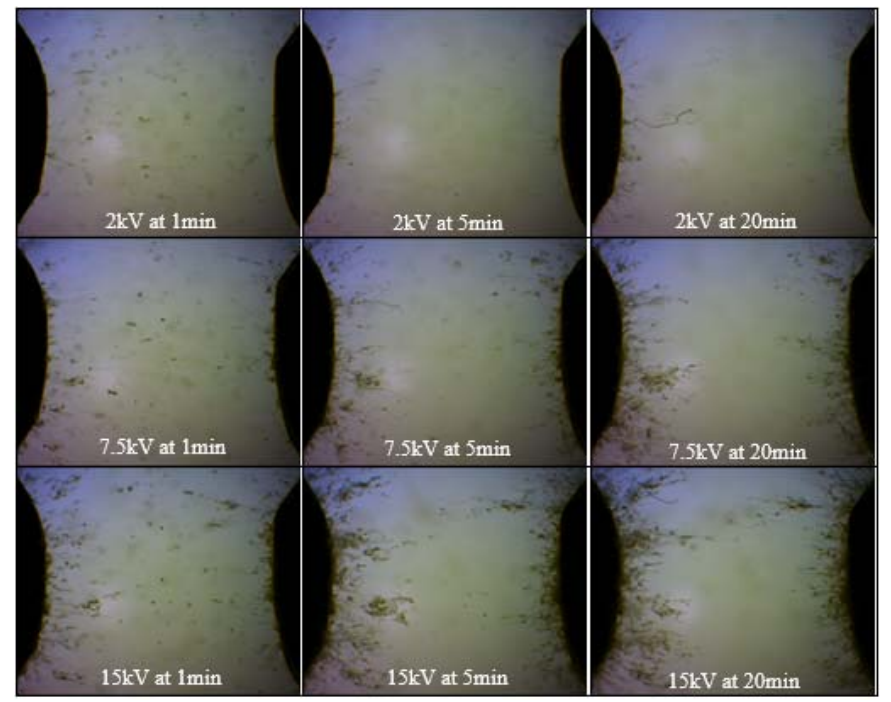

Fig. 3. Optical microscopic image of bridging for loose bonded covered electrode test under influence of DC electric field with $0.008 \%$ concentration of $150-250 \mu \mathrm{m}$ cellulose particles.

The graphs below in Fig. 4 shows the conduction current between the electrodes during the tests with different levels of contaminated transformer oil. There is a high polarization current observed for all the concentration levels then the current decayed slowly to steady state after around 600s. These currents did not have been influenced by contaminated particles as seen in the Fig. 4. It was observed from our previous tests that when there was a bridge between the 
electrodes, the conduction current increased almost linearly to level of contamination $[3,5]$.

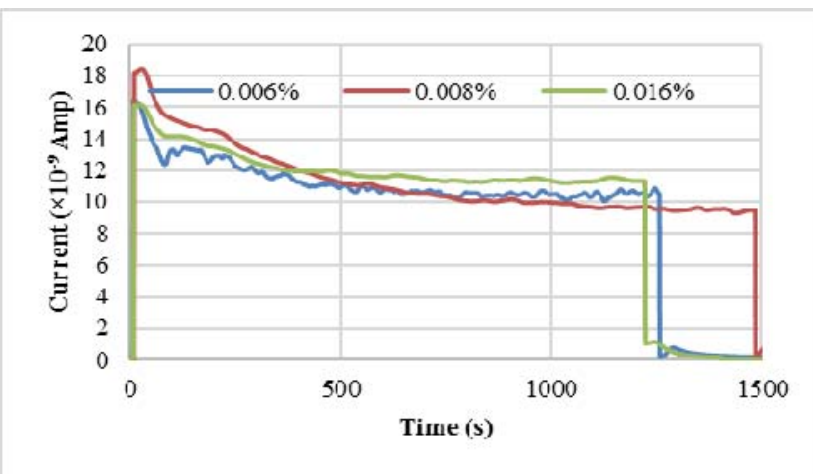

Fig. 4. Conduction currents with loosely bonded electrodes under the influence of $15 \mathrm{kV}$ DC electric fields with different concentration levels.

\section{B. Bridging with tight bond electrode}

Images from the tightly bonded kraft paper test are shown in Fig. 5. The pressboard particles started moving back and forth between the electrodes as soon as $1 \mathrm{kV}$ was applied. They were charging and discharging from one electrode to the other. They were initially drawing near the electrodes due to DEP force, once they touch the kraft paper covered electrode surface, they acquired charge from the surfaces. As the particles gathered charge, Coulomb force started to repel the particles from the electrodes due to same polarity. When repelling Coulomb force became greater than attracting DEP force, the particles finally started moving towards the other electrode. A detailed explanation of this process is given in [3]. The fibres started to attach on the paper cover of electrodes after a while. Initially the long fibres were attached to either of the electrodes and then the other small particles started attaching themselves to the elongated fibres. The bridging process started in this fashion and continues to grow. A very thin bridge formed for $1 \mathrm{kV}$ supply after $210 \mathrm{~s}$. There were few shallow bridges were created until 900s.

The movement of the particles along with bridging process intensified with the supplied voltage increased to $3 \mathrm{kV}$. A complete bridge was formed within 155s. The bridge between the electrodes were denser that previous test. The bridging process continued for 600s whereas no significant change observed.

The particles were moving between the electrodes more violently after the $6 \mathrm{kV}$ supply turned on. It took only 40 s to form the first thin bridge. The bridge became more strongly bonded and the thickness on the bridge was narrower in the milled and broadened towards the surface of the electrodes. Almost all the particles near the vicinity of the electrodes were drawn in to the bridge within 300 s.

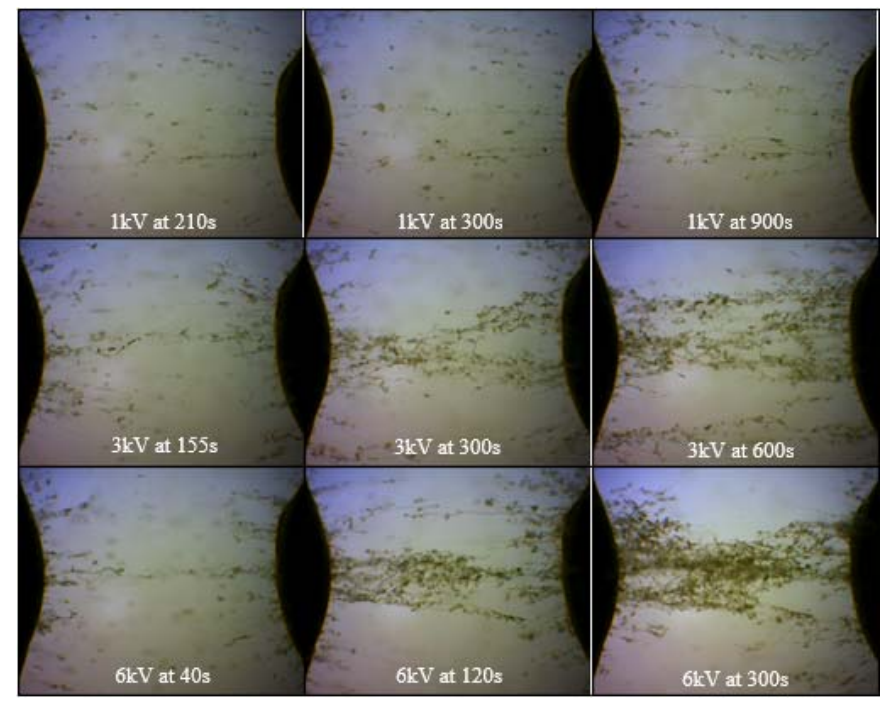

Fig. 5. Optical microscopic image of bridging for tight bonded covered electrode test under influence of DC electric field with $0.008 \%$ concentration of $150-250 \mu \mathrm{m}$ cellulose particles.

The bridging rate accelerated with increment of voltage (Fig. 5) as well as conduction current as shown in Fig. 6. The currents increased non-linearly with voltage.

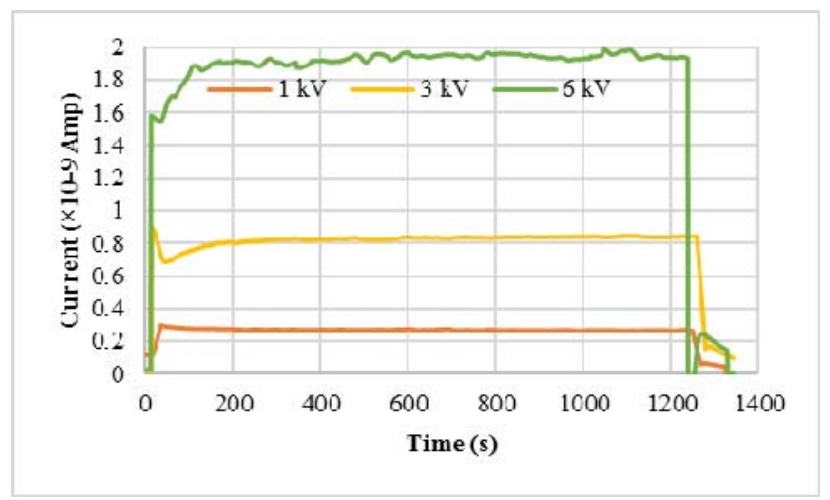

Fig. 6. Conduction currents with tight bonded electrodes under influence of different DC electric field with $0.008 \%$ concentration.

\section{Bridging with Paper Barrier}

The cellulose fibres started moving back and forward as soon as $2 \mathrm{kV}$ supply applied. They were charged by touching the bare electrodes and discharging to the paper barrier. Eventually the particles started to attach on the electrodes surface after a while. The bridging process starts with a longer fibre attached to the electrodes and starched towards other electrode. Then small particles attracted towards the long fibre. The bridge grows in this way from both electrodes to the paper wall. After $60 \mathrm{~s}$, a thin complete bridge formed to both side of the barrier and it continued to grow thicker until $600 \mathrm{~s}$ as shown in Fig. 7.

The pressboard fibres started moving more quickly after applying $7.5 \mathrm{kV}$. A thin complete bridge was observed within 20s. The bridge continued to grow thicker until 300s. There was no significant change observed after that. 
The particle movements were intensified rapidly for $15 \mathrm{kV}$. A thin complete bridge was formed within 10s from either side of the barrier. The bridge continued to grow thicker until 180 s whereby no apparent change observed.

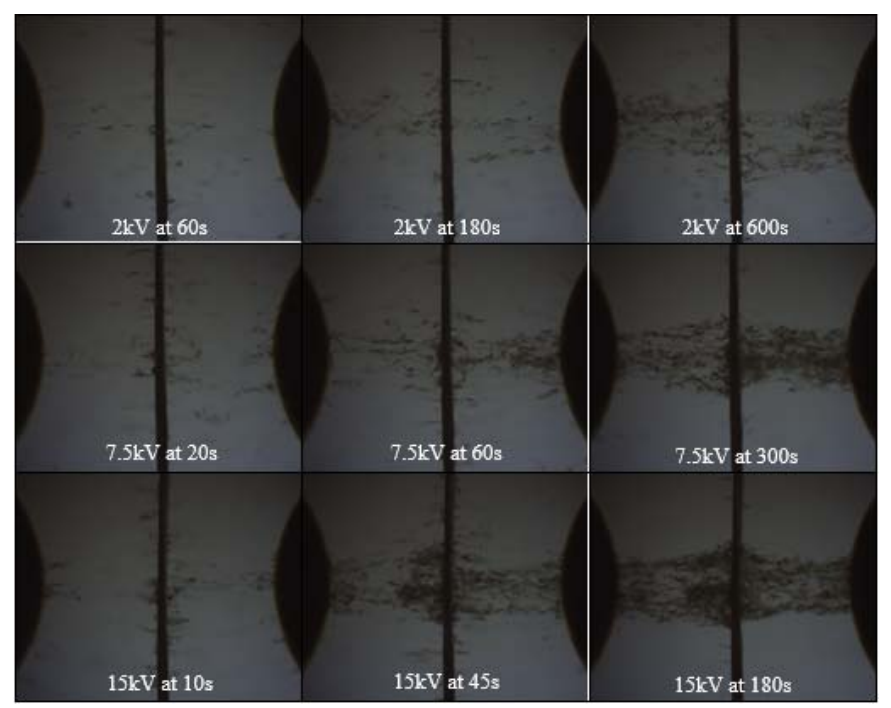

Fig. 7. Optical microscopic image of bridging for kraft paper barrier test under influence of DC electric field with $0.003 \%$ concentration of $150-250 \mu \mathrm{m}$ cellulose particles.

The conduction currents under the influence of $15 \mathrm{kV}$ DC electric field are shown in Fig. 8. The lowest current in the graph is for clean oil with the paper barrier inserted and rest of them are with different contaminant levels. All the contamination levels had a similar trend of conduction current. The currents increased almost linearly with the increment of particle contamination and the bridges were also thickened with it. The effects of the ion conduction in oil are not clear, but it probably plays a major role in clean oil. In the presence of contaminant particles the ions would end up on the particles which mobility is higher than the ions. So in contaminated oil the conduction is driven by the particles.

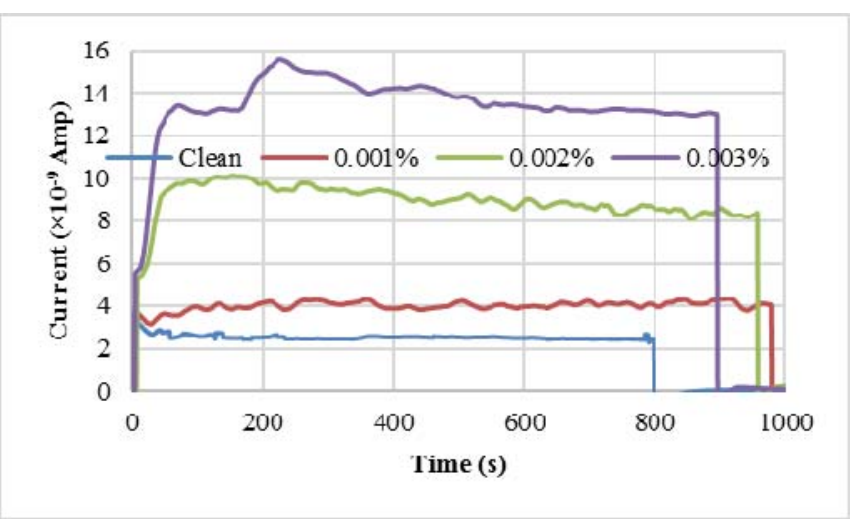

Fig. 8. Conduction currents with a paper barrier under influence of $15 \mathrm{kV}$ DC electric fields with clean oil and $0.003 \%$ contaminated oil.

\section{CONCLUSION}

There are several conclusions drawn from the above observations. In the case of the loose bond (with oil layer between electrodes' surface and the paper) there were no bridging observed. The dust particles stack to the paper and formed a beard without any bridge formation. It is concluded that the dust charging at the surfaces is the key mechanism for the bridging since it allows the particle to be detached from the electrodes surfaces by Coulomb force and to be transported to the central region between electrodes. The tight bond between electrodes' surface and the paper allows the charge transport to take place and the bridge is formed in this case. Moreover when a free-standing paper barrier is introduced between the electrodes, the dust particles acquire charges from the electrodes and transfer them to the paper barrier. Due to paper surface conduction this charge accumulates at particular points on the paper and plays an important role in consequent charging of the particles on the other side of the paper barrier, which in turn give a specific shape of the barrier. The conduction current with paper barrier is lower than the bridges without any barrier. So the paper barrier slows down the charge transportation process. On the other hand, the paper does not stop a charge transport through it completely, and multi-layered systems with oil-paper interleave arrangement would be preferable to stop bridging.

\section{Acknowledgment}

The authors acknowledge the project financial support received from IET Power Academy and the National Grid.

\section{REFERENCES}

[1] M.G. Danikas, "Breakdown of transformer oil". Electrical Insulation Magazine, IEEE. 6(5): p. 27-34, 1990.

[2] G. Chen and M. H. Zuber, "Pre-breakdown characteristics of contaminated power transformer oil". in Electrical Insulation and Dielectric Phenomena, 2007. CEIDP 2007. Annual Report - Conference on, 2007, pp. 659-662.

[3] S. Mahmud, G. Chen, I. O. Golosnoy, G. Wilson, \& P. Jarman, "Experimental Studies of influence of DC and AC Electric Fields on Bridging in Contaminated Transformer Oil". (submitted to IEEE TDEI)

[4] S. Mahmud, I. O. Golosnoy, G. Chen, G. Wilson, and P. Jarman, "Numerical simulations of bridging phenomena in contaminated transformer oil". Proceedings of 2012 IEEE Conference on Electrical Insulation and Dielectric Phenomena, Montreal, Canada, 14 - 17 Oct 2012.4pp, 383-386.

[5] S. Mahmud, G. Chen, I. O. Golosnoy, G. Wilson, and P. Jarman, "Bridging phenomenon in contaminated transformer oil". Proceedings of 2012 International Conference on Condition Monitoring and Diagnosis, Piscataway, US, Institute of Electrical and Electronics Engineers, 4pp, 180-183.

[6] S. Mahmud, G. Chen, I. O. Golosnoy, G. Wilson, \& P. Jarman, "Bridging in contaminated transformer oil under DC and AC electric field". Journal of Physics: Conference Series, 472(1), 12007, 2013.

[7] S. Mahmud, G. Chen, I. O. Golosnoy, G. Wilson, and P. Jarman, "Bridging in Contaminated Transformer Oil under AC, DC and DC Biased AC Electric Field". Proceedings of IEEE Conference on Electrical Insulation and Dielectric Phenomena, Shenzhen, China, 20 23, 2013. 